\title{
A Perturbation Method for Computing Field Distortions due to Conductive Regions with H-Conform Magnetodynamic Finite Element Formulations
}

\author{
Patrick Dular and Ruth V. Sabariego
}

\begin{abstract}
A method for solving eddy current problems in two separate steps is developed for global-local analyses with $h$ conform finite element formulations. An unperturbed problem is first solved in a global mesh excluding additional conductive regions. Its solution gives the sources for a sequence of other problems, perturbed by adding conductive regions. Each problem only requires a new adapted mesh of a local region. The way the local problems and their sources are defined leads to a significant speed-up of parameterized analyses, e.g. in optimization and sensitivity analyses.
\end{abstract}

Index Terms-Eddy currents, field distortion, finite element method, perturbation method.

\section{INTRODUCTION}

$\mathrm{R}$ epetitive finite element (FE) computations are needed when studying variations of geometrical and physical characteristics, what is the case for any parameterized analysis. It is worth then benefiting from previous computations instead of starting a new complete FE solution for any new variation.

When computing field distortions due to conductive regions (e.g., in magnetic shielding, eddy current non-destructive testing), an unperturbed solution followed by perturbed solutions due to additional conductive regions can be of great interest [1], [2]. Benefits are particularly aimed for allowing different problem-adapted meshes and for computational efficiency by decreasing the size of the problem.

A perturbation method for solving eddy current problems in two separate steps is developed for magnetic field $\boldsymbol{h}$ conform FE formulations. An unperturbed problem is first solved in a global domain excluding additional conductive regions and thus avoiding their mesh. Its solution gives the sources to supply perturbed problems when conductive regions are added. Volume sources are considered and compared to boundary sources. Their determination requires to project the solutions between discrete spaces (meshes) and adequate discretizations of the field quantities with edge FEs to allow the magnetic field conformity in both steps.

Application examples illustrate and validate the method, pointing out the parameters affecting the accuracy of the solution and the computational efficiency.

Manuscript received April 30,2006. This work was partly supported by the Belgian Science Policy (IAP P5/34) and the Belgian French Community (Research Concerted Action ARC 03/08-298).

P. Dular and R. V. Sabariego are with the University of Liège, Dept. of Electrical Engineering and Computer Science, B28, B-4000 Liège, Belgium (e-mail's: Patrick.Dular@ulg.ac.be, r.sabariego@ulg.ac.be). P. Dular is also with the Belgian National Fund for Scientific Research (F.N.R.S.).

\section{UNPERTURBED AND PERTURBED EDDY CURRENT PROBLEMS}

\section{A. Strong formulations}

Maxwell equations are to be solved in a bounded domain $\Omega$, with boundary $\partial \Omega$ (possibly at infinity), of the 2-D or 3-D Euclidean space. The eddy current conducting part of $\Omega$ is denoted $\Omega_{c}$ and the non-conducting one $\Omega_{c}{ }^{C}$, with $\Omega=\Omega_{c} \cup \Omega_{c}{ }^{C}$. Massive conductors belong to $\Omega_{c}$.

The equations and relations governing the magnetodynamic (eddy current) problem in $\Omega$ are

$$
\begin{gathered}
\operatorname{curl} \boldsymbol{h}=\boldsymbol{j}, \operatorname{curl} \boldsymbol{e}=-\partial_{\mathrm{t}} \boldsymbol{b}, \operatorname{div} \boldsymbol{b}=0, \\
\boldsymbol{b}=\mu \boldsymbol{h}, \boldsymbol{j}=\sigma \boldsymbol{e},
\end{gathered}
$$

where $\boldsymbol{h}$ is the magnetic field, $\boldsymbol{b}$ is the magnetic flux density, $\boldsymbol{e}$ is the electric field, $\boldsymbol{j}$ is the electric current density (including source and eddy currents), $\mu$ is the magnetic permeability and $\sigma$ is the electric conductivity.

An unperturbed problem is first defined in $\Omega$ without considering the properties of a so-called perturbing region $\Omega_{c, p} \subset \Omega_{c}$, which will further lead to field distortions. At the discrete level, this region is not described in the mesh of $\Omega$. The perturbed problem focuses thus on $\Omega_{c, p}$ and its neighborhood, their union $\Omega_{p}$ being adequately defined and meshed will serve as the studied domain. The subscripts $u$ and $p$ will refer to unperturbed and perturbed quantities, respectively. Fig. 1 illustrates the considered sequence of problems, pointing out the need of projecting fields from one mesh to another.
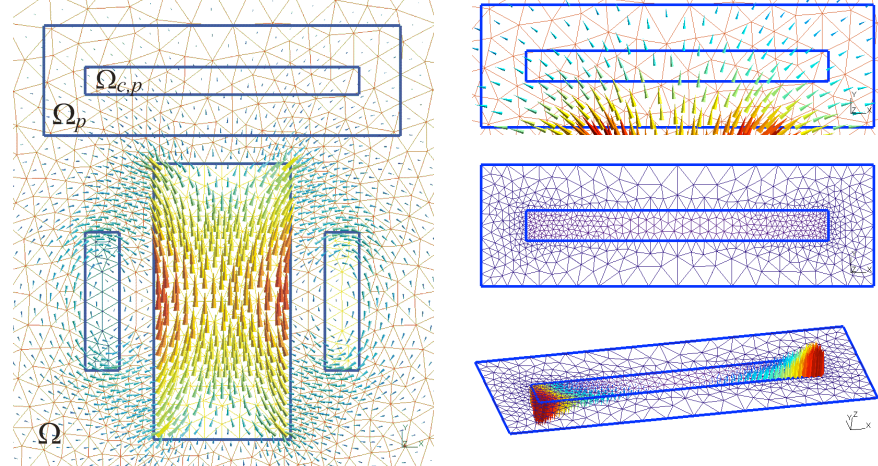

Fig. 1. Unperturbed (left: mesh of $\Omega$ and distribution of $\mu_{u} \boldsymbol{h}_{u}$ ) and perturbed problems (top right: $\mu_{u} \boldsymbol{h}_{u}$ to be projected in $\Omega_{c, p}$ for calculating a source field; middle right: adapted mesh of $\Omega_{p}$; bottom right: $\boldsymbol{j}=\operatorname{curl} \boldsymbol{h}$ in $\Omega_{c, p}$ ).

The addition of a perturbing conducting region $\Omega_{c, p}$ to the initial configuration gives rise to additional eddy currents and 
field distortions. The perturbed problem is defined as an eddy current problem in $\Omega_{p}$. Particularizing (1) and (2) for both the unperturbed and perturbed quantities, and subtracting the unperturbed equations from the perturbed ones, a perturbation problem (defined as the difference between perturbed and unperturbed problems) is obtained in $\Omega_{p}$ (initially in $\Omega$ ) [1], [2]. Keeping the equations in terms of the distortions $\boldsymbol{h}=\boldsymbol{h}_{p}$ $\boldsymbol{h}_{u}$ and $\boldsymbol{e}=\boldsymbol{e}_{p}-\boldsymbol{e}_{u}$, one gets

$$
\begin{gathered}
\operatorname{curl} \boldsymbol{h}=\sigma_{p} \boldsymbol{e}+\boldsymbol{j}_{s}, \\
\operatorname{curl} \boldsymbol{e}=-\mu_{p} \partial_{t} \boldsymbol{h}-\boldsymbol{k}_{s}, \\
\boldsymbol{n} \times\left.\boldsymbol{h}\right|_{\partial \Omega_{p}}=0 \text { or } \boldsymbol{n} \times\left.\boldsymbol{e}\right|_{\partial \Omega_{p}}=0,
\end{gathered}
$$

with the volume sources $\boldsymbol{j}_{s}$ and $\boldsymbol{k}_{s}$ defined only in $\Omega_{c, p}$ and given by the unperturbed solution, i.e.

$$
\begin{gathered}
\boldsymbol{j}_{S}=\left(\sigma_{p}-\sigma_{u}\right) \boldsymbol{e}_{u} \text { in } \Omega_{c, p}, \\
\boldsymbol{k}_{s}=\left(\mu_{p}-\mu_{u}\right) \partial_{t} \boldsymbol{h}_{u} \text { in } \Omega_{c, p} .
\end{gathered}
$$

The perturbation problem (3)-(7) is rigorously defined in the whole studied domain $\Omega$, taking account of the geometrical and material details of the initial unperturbed problem. The conditions $(5 \mathrm{a})$ or $(5 \mathrm{~b})$ neglect the distortion at a certain distance from $\Omega_{c, p}$, which is actually only correct at infinity (for $\Omega_{p}$ extended to the whole space). For convenience, an approximation neglecting some of these initial details will be made. The so-modified studied domain $\Omega_{p}$ can be a portion or not of $\Omega$, with or without inclusion of initial materials, these being possibly simplified. At the discrete level, the meshes of both unperturbed and perturbed problems can then be significantly simplified, each problem asking for mesh refinement of different regions.

\section{B. Sources of the perturbation problem}

The sources $\boldsymbol{j}_{s}$ and $\boldsymbol{k}_{S}(6)-(7)$ act as volume sources for the perturbation equations (3)-(4). This is a consequence of the use of the distortions $\boldsymbol{h}$ and $\boldsymbol{e}$ as unknowns instead of the perturbed fields $\boldsymbol{h}_{p}$ and $\boldsymbol{e}_{p}$ directly. Another implication is the homogeneous nature of the boundary conditions (5a) or (5b); (5a) should nevertheless be extended to a non-homogeneous condition (though independent of the perturbed solution) when a non-zero net perturbation current flows in $\Omega_{c, p}$.

The perturbed problem, with the unknown fields $\boldsymbol{h}_{p}$ and $\boldsymbol{e}_{p}$, would require non-homogeneous conditions such as

$$
\boldsymbol{n} \times\left.\boldsymbol{h}_{p}\right|_{\partial \Omega_{p}}=\boldsymbol{n} \times\left.\boldsymbol{h}_{u}\right|_{\partial \Omega_{p}} \text { or } \boldsymbol{n} \times\left.\boldsymbol{e}_{p}\right|_{\partial \Omega_{p}}=\boldsymbol{n} \times\left.\boldsymbol{e}_{u}\right|_{\partial \Omega_{p}} .
$$

The unperturbed fields would thus serve as surface sources, to be projected on the perturbed mesh boundary $\partial \Omega_{p}$. However, such conditions can only be applied on a finite boundary $\partial \Omega_{p}$. Indeed an infinite boundary would support a zero source, with no information at all for the perturbed problem. The unperturbed field $\boldsymbol{h}_{u}$ could alternatively be used as a volume source field in the whole $\Omega_{p}$, but with the disadvantage of needing its evaluation and projection on the whole domain.

These drawbacks justify the use of the volume sources $\boldsymbol{j}_{S}$ and $\boldsymbol{k}_{s}(6)-(7)$, the reduced support of which noticeably limits the evaluation and projection operations.

While the source $\mathbf{k}_{s}$ (7) can be determined from the known field $\boldsymbol{h}_{u}$, the source current density $\boldsymbol{j}_{S}(6)$ is to be obtained from the still undetermined unperturbed electric field $\mathbf{e}_{u}$. Indeed, in the considered case, with $\sigma_{u}=0$ and $\sigma_{p} \neq 0$ in $\Omega_{c, p}$, the field $\mathbf{e}_{u}$ is unknown in any non-conducting regions. This is an additional difficulty in comparison with the complementary case $\sigma_{u} \neq 0$ and $\sigma_{p}=0$ studied in [2]. The determination of $\mathbf{e}_{u}$ will require solving an electric problem defined by the Faraday and electric conservation equations, together with the electric constitutive relation.

\section{Magnetic Field CONFORM Weak Formulations}

\section{A. h-conform unperturbed solution as a source}

The conformity of $\boldsymbol{h}$ (conservation of its circulation or currents) can be assured through the definition of a magnetic scalar potential $\phi$ in $\Omega_{c}{ }^{C}$, with $\boldsymbol{h}=-\operatorname{grad} \phi$; this potential is discretized with nodal FEs. A total potential is multivalued when $\Omega_{c}{ }^{C}$ is multiply connected, case in which surface cuts must be defined to ensure a single valued potential. However a reduced potential can be continuously defined in the whole $\Omega_{c}{ }^{C}$ by means of source fields associated to each multiply connected portion of $\Omega_{c}$ [4]. In $\Omega_{c}$, the conformity of $\mathbf{h}$ is assured by properly defining its function space, through edge FEs at the discrete level to strongly express (1a).

The unperturbed field distribution is first calculated in $\Omega$ as the solution of an eddy current problem with a magnetic field conform FE formulation, obtained from the weak form of the Faraday equation (1b), i.e. [4],

$$
\begin{aligned}
\left(\partial_{t}\left(\mu_{u} \boldsymbol{h}_{u}\right), \boldsymbol{h}^{\prime}\right)_{\Omega} & +\left(\sigma_{u}^{-1} \operatorname{curl} \boldsymbol{h}_{u}, \operatorname{curl} \boldsymbol{h}^{\prime}\right)_{\Omega_{c} \backslash \Omega_{c, p}}+\left(\boldsymbol{e}_{u}, \operatorname{curl} \boldsymbol{h}^{\prime}\right)_{\Omega_{c, p}} \\
+ & <\boldsymbol{n} \times \boldsymbol{e}_{u}, \boldsymbol{h}^{\prime}>_{\Gamma_{e}}=0, \forall \boldsymbol{h}^{\prime} \in F^{1}(\Omega),
\end{aligned}
$$

where $F^{1}(\Omega)$ is the curl-conform function space defined on $\Omega$ and containing the basis functions for $\boldsymbol{h}_{u}$ as well as for the test function $\boldsymbol{h}^{\prime} ;(\cdot, \cdot)_{\Omega}$ and $\langle\cdot, \cdot\rangle_{\Gamma}$ respectively denote a volume integral in $\Omega$ and a surface integral on $\Gamma$ of the product of their vector field arguments. The surface term in (9) accounts for the natural boundary or interface conditions.

The volume integral term in $\Omega_{c, p}$ in (9), involving the electric field $\boldsymbol{e}_{u}$, is null at this step (test function $\boldsymbol{h}^{\prime}$ is curl-free because $\Omega_{c, p}$ is not yet subject to eddy currents). As a consequence, $\boldsymbol{e}_{u}$ in any portion of $\Omega_{c}{ }^{C}$ cannot be part of a single magnetodynamic solution. Its contribution in (9) will be further used by a perturbation problem (with non-curl-free $\boldsymbol{h}$ ). The unperturbed solution will act as a source for the perturbation problem.

\section{B. Repetitive perturbation eddy current problems}

For each added domain $\Omega_{c, p}$, the magnetic field conform FE formulation of the perturbation problem (3)-(7) is obtained as the weak form of the perturbation Faraday equation (4), together with the strong form of the perturbation Ampere equation (3), i.e.,

$$
\begin{aligned}
& \left(\partial_{t}\left(\mu_{p} \boldsymbol{h}\right), \boldsymbol{h}^{\prime}\right)_{\Omega_{p}}+\left(\boldsymbol{k}_{s}, \boldsymbol{h}^{\prime}\right)_{\Omega_{c, p}}+\left(\sigma_{p}^{-1} \operatorname{curl} \boldsymbol{h}, \operatorname{curl} \boldsymbol{h}^{\prime}\right)_{\Omega_{c, p}} \\
& -\left(\sigma_{p}^{-1} \boldsymbol{j}_{S}, \operatorname{curl} \boldsymbol{h}^{\prime}\right)_{\Omega_{c, p}}+<\boldsymbol{n} \times \boldsymbol{e}, \boldsymbol{h}^{\prime}>_{\Gamma_{e}}=0, \forall \boldsymbol{h}^{\prime} \in F^{1}\left(\Omega_{p}\right) .
\end{aligned}
$$

At the discrete level, the source quantities $\boldsymbol{j}_{S}$ and $\boldsymbol{k}_{S}$ initially given in the unperturbed mesh have to be expressed in the 
perturbed one. This can be done through a projection method [5] with target quantities $\boldsymbol{j}_{S}$ and $\boldsymbol{k}_{S}$ defined in adequate function spaces. From (3), the projected $\boldsymbol{j}_{S}$ should have the same conformity as curl $\boldsymbol{h}$, while from (4), the projected $\boldsymbol{k}_{s}$ should have the same conformity as $\boldsymbol{h}$. The field $\boldsymbol{k}_{S}(7)$ can be directly obtained via the projection of $\boldsymbol{h}_{u}$. However the field $\boldsymbol{j}_{s}(6)$ requires an intermediate step for obtaining $\boldsymbol{e}_{u}$, as developed hereafter.

\section{Electric problem in added conducting regions}

The unperturbed electric field $\mathbf{e}_{u}$ is to be determined in the new added perturbing conducting region $\Omega_{c, p}$ to express the source quantity $\boldsymbol{j}_{S}(6)$ in (3) or (10). It can be calculated via an electric problem defined in $\Omega_{c, p}$ by

$$
\operatorname{curl} \boldsymbol{e}_{u}=-\partial_{\mathrm{t}}\left(\mu_{u} \boldsymbol{h}_{u}\right), \operatorname{div} \boldsymbol{d}_{u}=0, \boldsymbol{d}_{u}=\varepsilon_{u} \boldsymbol{e}_{u},(11 \mathrm{a}-\mathrm{b}-\mathrm{c})
$$

where the newly defined quantities are the unperturbed electric flux density $\boldsymbol{d}_{u}$ and the electric permittivity $\varepsilon_{u}$. Equation (11b) assumes that no charge density exists in $\Omega_{c, p}$.

The required conformity of $\boldsymbol{e}_{u}$ is the one of curl $\boldsymbol{h}$, i.e. of a curl-field. This can be satisfied through the definition of an electric vector potential $\boldsymbol{u}$ as primal unknown field, with

$$
\boldsymbol{d}_{u}=\operatorname{curl} \boldsymbol{u},
$$

thus satisfying (11b). The electric model is then governed by the weak form of (11a) in the sub-domain $\Omega_{c, p}$, i.e. an electric flux density conform formulation [3],

$$
\begin{aligned}
& \left(\varepsilon_{u}^{-1} \operatorname{curl} \boldsymbol{u}, \operatorname{curl} \boldsymbol{u}^{\prime}\right)_{\Omega_{c, p}}+\left(\partial_{t}\left(\mu_{u} \boldsymbol{h}_{u}\right), \boldsymbol{u}^{\prime}\right)_{\Omega_{c, p}} \\
& +<\boldsymbol{n} \times \boldsymbol{e}_{u}, \boldsymbol{u}^{\prime}>_{\partial \boldsymbol{\Omega}_{c, p}}=0, \forall \boldsymbol{u}^{\prime} \in F^{1}\left(\boldsymbol{\Omega}_{c, p}\right),
\end{aligned}
$$

where the function space $F^{1}\left(\Omega_{c, p}\right)$ contains $\boldsymbol{u}$ and its associated test function $\boldsymbol{u}^{\prime}$ and has to be constrained with a gauge condition. At the discrete level, $\boldsymbol{u}$ is discretised with edge FEs and is associated a gauge condition by the tree cotree technique.

The electric model to be posed in $\Omega_{c, p}$ gets information regarding the tangential electric field on $\partial \Omega_{c, p}$ via a natural boundary condition that appears in the surface integral term of (13). Its expression for each test function $\boldsymbol{u}^{\prime}$ can be directly given by (9) written only for $\Omega_{p} \backslash \Omega_{c, p}$ (this is a consequence of the similar conformity of $\boldsymbol{u}$ and $\boldsymbol{h}_{u}$ ), i.e.,

$$
\begin{gathered}
\left(\partial_{t}\left(\mu_{u} \boldsymbol{h}_{u}\right), \boldsymbol{u}^{\prime}\right)_{\Omega_{p} \backslash \Omega_{c, p}}+\left(\sigma_{u}^{-1} \operatorname{curl} \boldsymbol{h}_{u}, \operatorname{curl} \boldsymbol{u}^{\prime}\right)_{\Omega_{c} \backslash \Omega_{c, p}} \\
+<\boldsymbol{n} \times \boldsymbol{e}_{u}, \boldsymbol{u}^{\prime}>_{\partial \Omega_{c, p}}=0
\end{gathered}
$$

Adding (13) and (14), with normal vectors $\boldsymbol{n}$ exterior to $\Omega_{c, p}$ and $\Omega_{p} \backslash \Omega_{c, p}$ respectively, thus of opposite signs, the following equation is obtained

$$
\begin{gathered}
\left(\varepsilon_{u}^{-1} \operatorname{curl} \boldsymbol{u}, \operatorname{curl} \boldsymbol{u}^{\prime}\right)_{\Omega_{c, p}}+\left(\partial_{t}\left(\mu_{u} \boldsymbol{h}_{u}\right), \boldsymbol{u}^{\prime}\right)_{\Omega_{c, p}} \\
+\left(\partial_{t}\left(\mu_{u} \boldsymbol{h}_{u}\right), \boldsymbol{u}^{\prime}\right)_{\Omega_{p} \backslash \Omega_{c, p}}+\left(\sigma_{u}^{-1} \operatorname{curl} \boldsymbol{h}_{u}, \operatorname{curl} \boldsymbol{u}^{\prime}\right)_{\Omega_{c} \backslash \Omega_{c, p}}=0 \\
\forall \boldsymbol{u}^{\prime} \in F^{1}\left(\Omega_{c, p}\right) .
\end{gathered}
$$

Equation (15) illustrates well how $\boldsymbol{h}_{u}$ acts as a source for determining $\boldsymbol{u}$ in $\Omega_{c, p}$ and $\partial \Omega_{c, p}$, both as a volume source $\partial_{\mathrm{t}}\left(\mu_{u} \boldsymbol{h}_{u}\right)$ by (11a) and a boundary source naturally converted to a volume source by (14) (via a volume integration limited to the layer of FEs touching $\partial \Omega_{c, p}$ in $\left.\Omega_{p} \backslash \Omega_{c, p}\right)$. At the discrete level, the field $\boldsymbol{h}_{u}$ is projected from its initial mesh to that of $\Omega_{c, p}$ before being used in (15).

As a result, using (6), $\sigma_{u}=0,(11 \mathrm{c})$ and (12), the source quantity $\boldsymbol{j}_{S}$ is obtained with the desired conformity, i.e.,

$$
\boldsymbol{j}_{S}=\sigma_{p} \boldsymbol{e}_{u}=\sigma_{p} \varepsilon_{u}^{-1} \boldsymbol{d}_{u}=\sigma_{p} \varepsilon_{u}^{-1} \operatorname{curl} \boldsymbol{u}
$$

\section{Back to the eddy current perturbation problems}

The source quantities $\boldsymbol{k}_{s}(7)$ and $\boldsymbol{j}_{s}(16)$ can then be used in (10), the solution of which gives the eddy current density in $\Omega_{c, p}$ and the ensuing field distortions.

\section{E. Impedance variation of source inductors}

Similarly to what was demonstrated in [2], the use of the sources (6) and (7) constitutes an efficient way to calculate the impedance changes of coils of the unperturbed problem, which is of interest e.g. in non-destructive applications.

A suitable treatment of the surface integral term in (9) for an inductor consists in naturally defining a global voltage $V_{u}$ in a weak sense. A global test function for $\boldsymbol{h}_{u}$ can be defined with a unit circulation along any current tube of the inductor so that the surface integral in (9) can be expressed as the product of a global voltage $V_{u}$ and a unit global current I( $\left.\operatorname{curl} \boldsymbol{h}^{\prime}\right)$ [4].

Choosing as test functions $\boldsymbol{h}^{\prime}=\boldsymbol{h}_{p}$ in the unperturbed formulation (9) and $\boldsymbol{h}^{\prime}=\boldsymbol{h}_{u}$ in the perturbed one, and subtracting the former from the latter, one gets the perturbation voltage $V$ as follows

$$
\begin{aligned}
& \left(\partial_{t}\left(\mu_{p} \boldsymbol{h}_{p}\right), \boldsymbol{h}_{u}\right)_{\Omega}+\left(\sigma_{p}^{-1} \operatorname{curl} \boldsymbol{h}_{p}, \operatorname{curl} \boldsymbol{h}_{u}\right)_{\Omega_{c} \backslash \Omega_{c, p}} \\
& +\left(\sigma_{p}^{-1} \operatorname{curl} \boldsymbol{h}_{p}, \operatorname{curl} \boldsymbol{h}_{u}\right)_{\Omega_{c, p}}-\left(\partial_{t}\left(\mu_{u} \boldsymbol{h}_{u}\right), \boldsymbol{h}_{p}\right)_{\Omega} \\
& -\left(\sigma_{u}^{-1} \operatorname{curl} \boldsymbol{h}_{u}, \operatorname{curl} \boldsymbol{h}_{p}\right)_{\Omega_{c} \backslash \Omega_{c, p}}-\left(\boldsymbol{e}_{u}, \operatorname{curl} \boldsymbol{h}_{p}\right)_{\Omega_{c, p}} \\
& =V_{p} I\left(\operatorname{curl} \boldsymbol{h}_{u}\right)-V_{u} I\left(\operatorname{curl} \boldsymbol{h}_{p}\right)=V .
\end{aligned}
$$

With $\mu_{p}=\mu_{u}$ and $\sigma_{p}=\sigma_{u}$ in $\Omega \backslash \Omega_{c, p}, \operatorname{curl} \boldsymbol{h}_{u}=0$ in $\Omega_{c, p}$ (7) and $\boldsymbol{h}=\boldsymbol{h}_{p}-\boldsymbol{h}_{u}$, (17) becomes

$$
\begin{aligned}
V=-\left(\boldsymbol{e}_{u}, \operatorname{curl} \boldsymbol{h}\right)_{\Omega_{c, p}}+\left(\boldsymbol{k}_{s}, \boldsymbol{h}_{u}\right)_{\Omega_{c, p}} & \\
& +\left(\partial_{t}\left(\mu_{p} \boldsymbol{h}\right), \boldsymbol{h}_{u}\right)_{\Omega_{c, p}}-\left(\partial_{t}\left(\mu_{u} \boldsymbol{h}_{u}\right), \boldsymbol{h}\right)_{\Omega_{c, p}} .
\end{aligned}
$$

The perturbation voltage calculation, and, in the frequency domain, the ensuing impedance variation $Z$ (the current $I$ in the inductor is fixed for both unperturbed and perturbed problems, thus $Z=V / I$ ), can thus be calculated via (18) by integrating some contributions only in $\Omega_{c, p}$. At the discrete level, this will give appreciable advantages by avoiding the projection of the perturbed solution to its initial source inductor, with the risk of a strong influence of the discretization error.

\section{APPLICATION}

An inductor core system (Fig. 1) is considered as a 2-D test problem to illustrate and validate the whole perturbation procedure (coil with 1000 turns, current $1 \mathrm{~A}$, frequency $50 \mathrm{~Hz}$, relative permeability $\mu_{r \text {, core }}=100$, core size $100 \mathrm{~mm} \times$ $200 \mathrm{~mm})$. The perturbing conductive region $\Omega_{c, p}$ is a rectangular plate $\left(\sigma_{\text {plate }}=5.910^{7} \Omega^{-1} \mathrm{~m}^{-1}, \mu_{r \text {, plate }}=1\right.$, size 
$100 \mathrm{~mm} \times 20 \mathrm{~mm})$.

Fig. 2 shows examples of meshes for the unperturbed and perturbation problems. Fig. 3 illustrates the sequence of associated solutions to be considered with the developed perturbation method. The local perturbation fields have been checked to be very similar to these obtained with the classical FE technique.

The coil impedance variation versus the distance separating the source and perturbing regions is depicted in Fig. 4 for different extensions of the perturbation domain. The reference solution is calculated from the conventional FE technique, subtracting the unperturbed solution from the perturbed one (with the major drawback that the same mesh is to be used for both problems to have the same discretization error, usually much higher than the impedance variation). Note that the dissipated Joule power in $\Omega_{c, p}$ is directly linked to the real part of the impedance variation (see (18) with $\mu_{u}=\mu_{p}$ ). The interest of extending the studied perturbation domain $\Omega_{p}$ up to infinity, through a transformation technique [6], [7], is pointed out, which justifies the preference for perturbation boundary conditions at infinity.

The difference with the reference solution increases with the decreasing distance between the regions what highlights a significant coupling of these regions. A more accurate solution for close positions would need an iterative procedure to calculate successive perturbations in each region, not only from the initial source region to the added plate but also from the latter to the former.
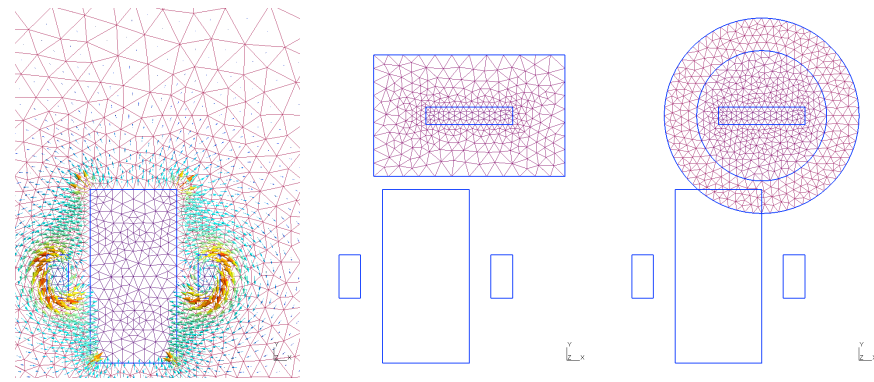

Fig. 2. Meshes of the unperturbed problem (left; with field $\boldsymbol{h}_{u}$ ) and perturbation problems with finite (middle) and infinite (right; [6], [7]) boundaries. Any intersection of perturbation boundaries (or $\Omega_{p} \backslash \Omega_{c, p}$ ) with the unperturbed problem material regions is allowed.
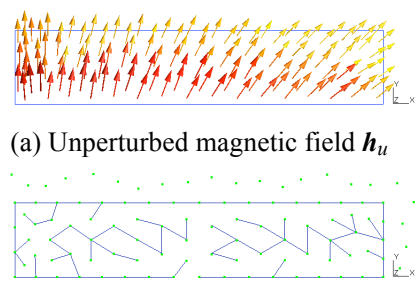

(c) Tree of edges in $\Omega_{c, p}$

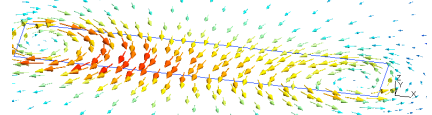

(e) Perturbation magnetic field $\boldsymbol{h}$ (a) Unperturbed magnetic field $\boldsymbol{h}_{u}$

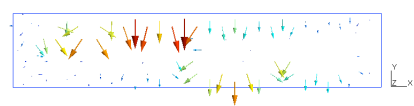

(b) Electric vector potential $\boldsymbol{u}$

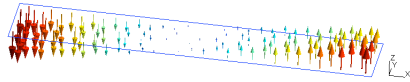

(d) Unperturbed electric field $\boldsymbol{e}_{u}$

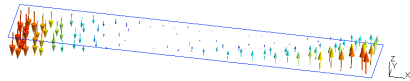

(f) Eddy current density $\boldsymbol{j}=\operatorname{curl} \boldsymbol{h}$
Fig. 3. The unperturbed field $\boldsymbol{h}_{u}$ projected in $\Omega_{c, p}$ (a) is a volume source for the electric problem, the solution of which is the electric vector potential $\boldsymbol{u}$ (b), gauged by the tree co-tree technique (tree of edges (c)), and the ensuing unperturbed electric field $\boldsymbol{e}_{u}=\varepsilon_{u}{ }^{-1}$ curl $\boldsymbol{u}(\mathrm{d})$. The solution of the perturbation problem is $\boldsymbol{h}$ (e) and $\boldsymbol{j}=\operatorname{curl} \boldsymbol{h}$ in $\Omega_{c, p}(\mathrm{f})$.

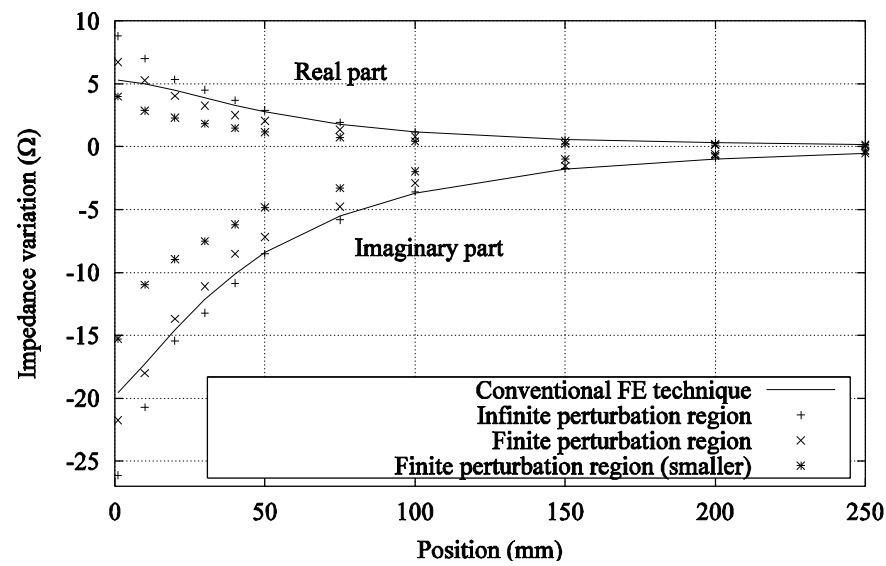

Fig. 4. Coil impedance variation $Z$ versus the distance separating the source region and the perturbing one $\Omega_{c, p}$.

For the considered problem, a sequence of 100 perturbation solutions has been solved with a speed-up factor of 30 in comparison to the conventional FE technique.

\section{CONCLUSIONS}

A method has been developed for efficiently exploiting the solution of a magnetodynamic FE problem as a source for other problems with added conductive materials. Each problem uses an adapted mesh, which necessitates projections of fields between them. The advantages of defining the induced electric field, solution of an electric problem, as a volume source limited to the added regions have been pointed out. These mainly concern the boundary conditions possibly defined at infinity, the reduced computational efforts for evaluating and projecting the sources, as well as the calculation of the impedance variations. As a consequence, a significant speed-up of parameterized analyses can be obtained, e.g. in optimization and sensitivity analyses. Benefits could also be obtained for moving systems.

\section{REFERENCES}

[1] Z. Badics et al., "An effective 3-D finite element scheme for computing electromagnetic field distorsions due to defects in eddy-current nondestructive evaluation", IEEE Trans. Magn., Vol. 33, No. 2, pp. 1012-1020, 1997.

[2] R. V. Sabariego and P. Dular, "A perturbation technique for the finite element modelling of nondestructive eddy current testing", in Proc. International Symposium on Electromagnetic Fields in Mechatronics, Electrical and Electronic Engineering (ISEF), Sept. 15-17, 2005.

[3] P. Dular and P. Kuo-Peng, "Dual finite element formulations for the three-dimensional modeling of both inductive and capacitive effects in massive inductors", IEEE Trans. Magn., Vol. 42, No. 4, pp. 743-746, 2006.

[4] P. Dular, P. Kuo-Peng, C. Geuzaine, N. Sadowski, J.P.A. Bastos, "Dual magnetodynamic formulations and their source fields associated with massive and stranded inductors", IEEE Trans. Magn., Vol. 36, No. 4, pp. 3078-3081, 2000.

[5] C. Geuzaine, B. Meys, F. Henrotte, P. Dular, W. Legros, "A Galerkin projection method for mixed finite elements", IEEE Trans. Magn., Vol. 35, No. 3, pp. 1438-1441, 1999.

[6] J.F. Imhoff, G. Meunier, X. Brunotte, J.C. Sabonnadière, "An original solution for unbounded electromagnetic 2D and 3D problems throughout the finite element method", IEEE Trans. Magn., Vol. 26, No. 5, pp. 1629$1631,1990$.

[7] F. Henrotte, B. Meys, H. Hedia, P. Dular, W. Legros, "Finite element modelling with transformation techniques", IEEE Trans. Magn., Vol. 35, No. 3, pp. 1434-1437, 1999. 